\title{
Comparison of ATP Simulation and Micro- processor Based Fault Location Using DFT
}

\author{
H Nouri ${ }^{*}$, F Jalili ${ }^{2}$, T Boxshall ${ }^{3}$ \\ Power Systems, Electronics and Control Research Laboratory, UWE Bristol, UK 1\&3, Sadid Pipe \& Equipment Co, \\ Saveh Road, Tehran, Iran² \\ ${ }^{* 1}$ hassan.nouri@uwe.ac.uk
}

\begin{abstract}
This paper evaluates the results of a microprocessor based fault locator employed on a laboratory power system model with those obtained within EMTP/ATP simulations. The Discrete Fourier Transform concept is used to extract the fundamental frequency components of the voltage and current waveforms under fault and the reactance based method to locate the fault. The results suggest a close correlation between the experimental and simulation studies for all fault types.
\end{abstract}

Keywords

Fault Location; Discrete Fourier Transform; Microprocessor; EMTP/ATP

\section{Introduction}

Distribution line faults have been a primary concern to networks. Faults affect the reliability of the service to the customer, the stability of the power system and the quality of the power delivered. Distribution line faults can even cause complete supply outages. These faults must be detected quickly for fast deactivation of the faulty section of line, in order to reroute the power to the customer to meet target restoration times.

Distribution line fault locators can be classified under two categories. The first is the impedance based locators, which analyse the power frequency $(50 \mathrm{~Hz}$ or $60 \mathrm{~Hz}$ ). The second is the travelling wave based locators which analyse the travel time of high frequency transients. Transient based fault location methods require higher sampling rates and more responsive voltage or current transducers. They also require multiple fault transient detectors placed throughout the system, which are clocked using GPS technology and data sent to a central location via a communication link. The use of GPS can make such a solution very expensive. Transient based fault location techniques for radial, meshed and distribution lines with tapped load power system configurations are described by a number of researchers (Nouri et al 2014, Magnago et al 1998 and Nouri et al 2001), respectively. In impedance based methods, the ratio of extracted fundamental frequency of the voltage and current waveforms yields the impedance seen from the generator up to the point of fault. A useful summary of the available impedance calculations can be found in (Zimmerman 2005). The reactance equation that will be used to obtain the fault location $(\mathrm{m})$ in per unit is expressed as:

$$
m=\frac{I M\left\{\frac{v_{s}}{i_{s}}\right\}}{X_{1 T L}}
$$

where IM defined as storing of the imaginary part of the voltage $\left(v_{s}\right)$ and the current $\left(i_{s}\right)$ ratio at the sending end of the line, and $X_{1 T L}$ is the positive sequence reactance of the distribution line.

\section{Discrete Fourier Transform}

\section{Mathematical Background}

In power systems the voltage and current waveforms are essentially shifted sine or cosine waves of fundamental frequency. These signals are effectively periodic when the system is running under steady state conditions. Under fault conditions, the fundamental frequency of the voltage and current amplitude can be extracted from the non 
periodic signals using the Fourier Transform. The basis functions chosen for the Fourier Transform is the complex exponential $g(t)=e^{-j \omega t}$. The Fourier Transform is defined as follows:

$$
F(\omega)=\int_{-\infty}^{\infty} f(t) e^{-j \omega t} d t
$$

On closer examination of (2), it can be seen that Fourier Transform integration takes place over all of time, i.e. from $-\infty$ to $\infty$. Therefore, the Fourier Transform should be reserved for periodic signals only. A power system contains many devices such as transformers, loads and switching circuits. These all create harmonic distortion under steady state conditions. The Fourier Transform is useful to analyse the harmonic content of the signal. However, when the system is running under a fault condition, non periodic signals exist. Consequently, the Fourier Transform should not be used to analyse fault conditions. An alternative form of Fourier's analysis techniques should be used, known as the Fourier Series. Unlike the Fourier Transform, the Fourier Series analyses the signal over a finite period of time. The basic assumption used in Fourier Series is that the waveform that results from the fault condition is assumed to be periodic within the interval from $t_{0}$ to $t_{0}+T$, where $T$ is the period of the fundamental component. The following equations are derived from (Wood 1984, PhadKe et al 1998, Johns et al 1995, and Croft et al 2001).

The voltage or current waveform $s(t)$ can be represented using the Fourier Series.

$$
s(t)=\frac{a_{0}}{2}+\sum_{n=1}^{\infty} a_{n} \cos n \omega_{0} t+\sum_{n=1}^{\infty} b_{n} \sin n \omega_{0} t
$$

where the Fourier coefficients are:

$$
a_{n}=\frac{2}{T} \int_{t_{0}}^{t_{0}+T} s(t) \cos n \omega_{0} t d t
$$

And

$$
b_{n}=\frac{2}{T} \int_{t_{0}}^{t_{0}+T} s(t) \sin n \omega_{0} t d t
$$

where $n=1,2,3 \ldots$, for the fundamental, second, and third harmonic ... . It is apparent that the fundamental frequency can be selected by setting $n=1$. Thus, the Fourier coefficients for the fundamental frequency can be determined:

$$
a_{1}=\frac{2}{T} \int_{t_{0}}^{t_{0}+T} s(t) \cos \omega_{0} t d t
$$

And

$$
b_{1}=\frac{2}{T} \int_{t_{0}}^{t_{0}+T} s(t) \sin \omega_{0} t d t
$$

In discrete analysis the time of the jth sample is $t_{j}=j \Delta t$ and the period of the waveform is $T=N \Delta t$, where $N$ is the number of samples per cycle of $50 \mathrm{~Hz}$. Now (6) and (7) can be determined in discrete time:

$$
a_{1}=\frac{2}{N \Delta t} \sum_{j=0}^{N-1} s\left(t_{j}\right) \cos \omega_{0} t_{j}=\frac{2}{N \Delta t} \sum_{j=0}^{N-1} s(j \Delta t) \cos \omega_{0} j \Delta t=\frac{2}{N} \sum_{j=0}^{N-1} s(j) \cos \omega_{0} j \Delta t
$$

And

$$
b_{1}=\frac{2}{N \Delta t} \sum_{j=0}^{N-1} s\left(t_{j}\right) \sin \omega_{0} t_{j}=\frac{2}{N \Delta t} \sum_{j=0}^{N-1} s(j \Delta t) \sin \omega_{0} j \Delta t=\frac{2}{N} \sum_{j=0}^{N-1} s(j) \sin \omega_{0} j \Delta t
$$

The amplitude of the real and imaginary parts of the fundamental frequency signal are represented by the Fourier coefficients $a_{1}$ and $b_{1}$ respectively. It is necessary to determine the amplitude of the fundamental frequency in order to apply an impedance equation to locate the fault, 


$$
|s|=\sqrt{a_{1}^{2}+b_{1}^{2}}
$$

Also it is required to find the phase angle between the real and imaginary components of the fundamental frequency,

$$
\angle s=\tan ^{-1}\left(\frac{b_{1}}{a_{1}}\right)
$$

\section{Practical Application of DFT}

A single line diagram of the laboratory power system model is shown in Fig. 1, with a fault locator system connected to bus-A. It can be assumed that the distribution line has a length of $1 \mathrm{pu}$.

A system must be designed to gather the phase voltages and currents and upload them to the CPU to analyse the fault conditions. This requires a sensor circuit to measure the voltages and currents, interfaces between the analogue and digital domains, and an external microprocessor to control the data gathering process. The chosen external microprocessor is the Siemens C164CI on the PHYTEC kitCON-164 evaluation board. A block diagram of the required system is shown in Fig. 2. The ADC and DAC are 12 bit devices. The data sent between the C164CI microcontroller and the CPU is via an RS-232 bus and the method for the $\mathrm{C} 164 \mathrm{CI}$ communicating with the ADC and DAC is a serial peripheral interface (SPI) bus.

A photograph of the laboratory power system model and microprocessor based fault locator is shown in Fig. 3.

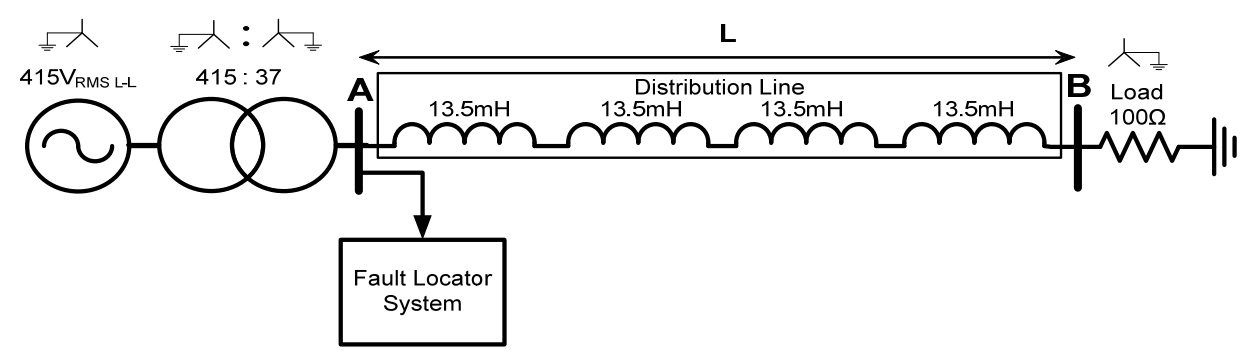

FIG. 1 SINGLE LINE DIAGRAM OF THE LABORATORY POWER SYSTEM MODEL

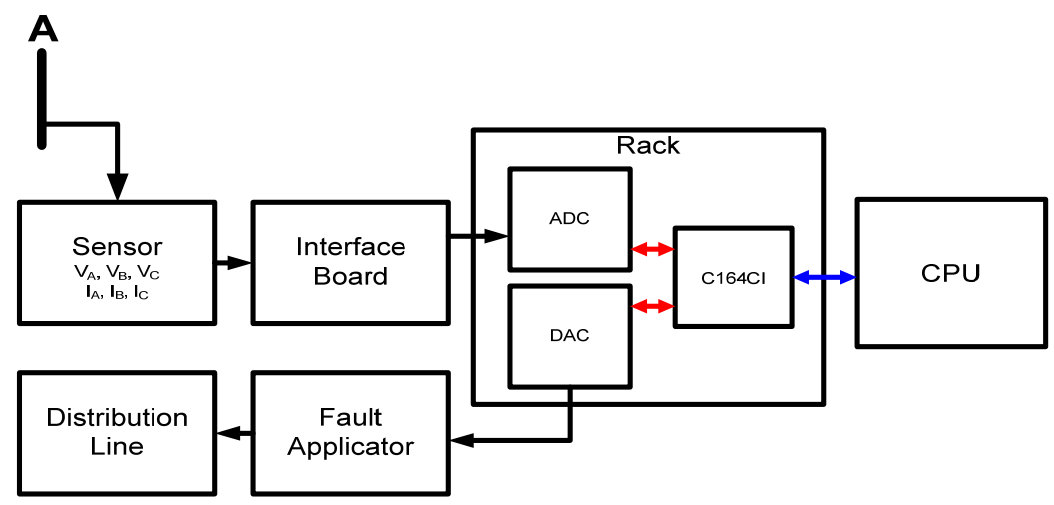

FIG. 2 A BLOCK DIAGRAM OF THE DATA ACQUISITION SYSTEM

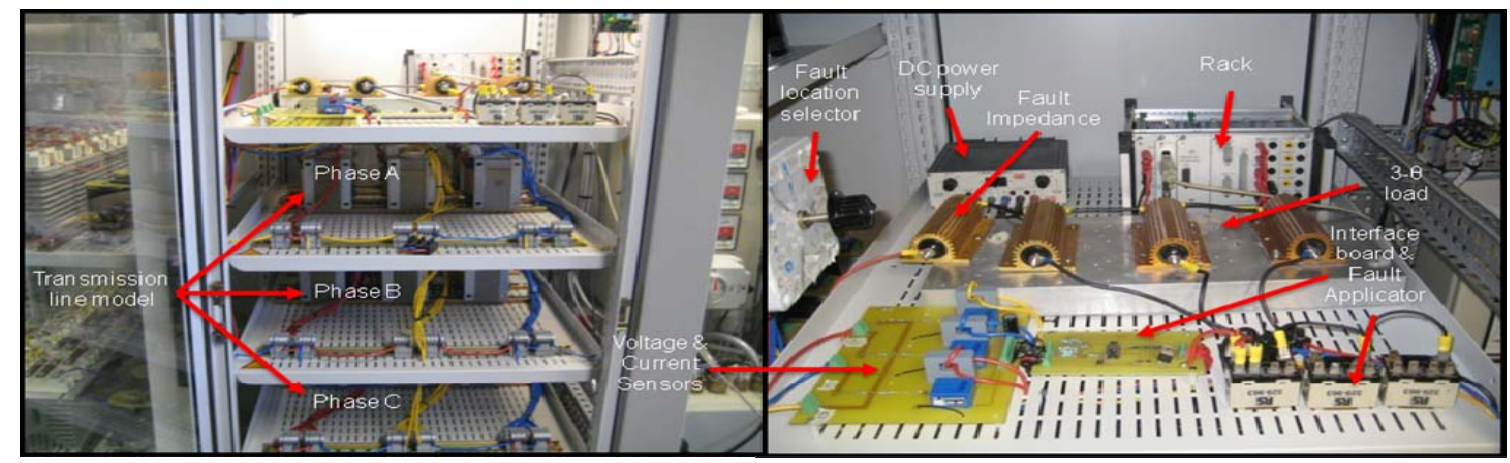

FIG. 3 PHOTOGRAPH OF LABORATORY POWER SYSTEM MODEL AND MICROPROCESSOR FAULT LOCATOR 


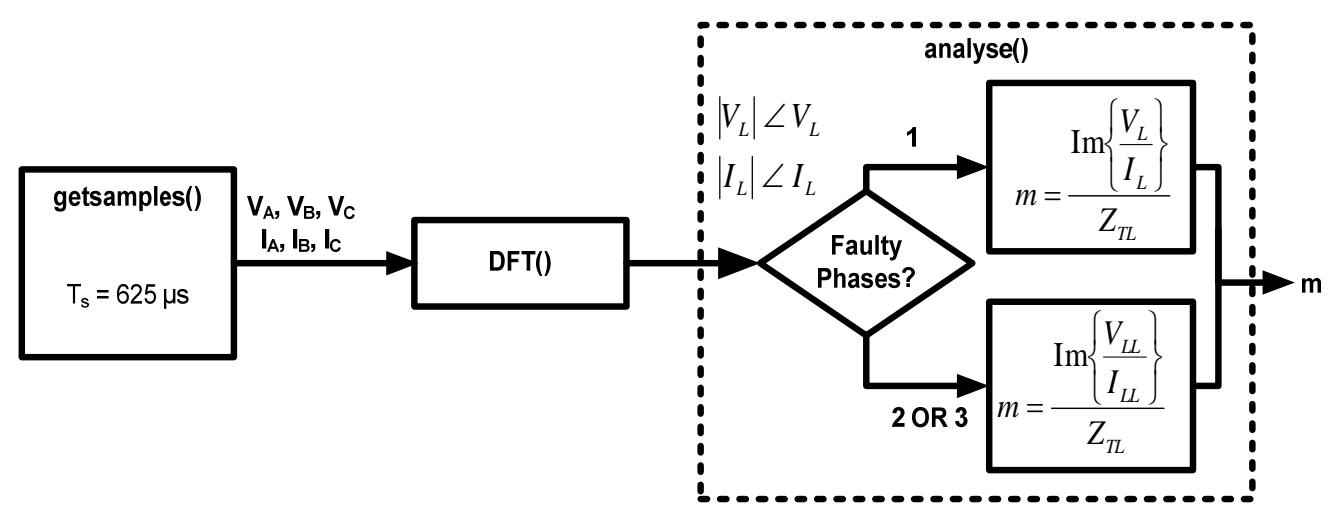

FIG. 4 BLOCK DIAGRAM OF SOFTWARE FUNCTIONALITY

\section{Fault Location Software}

The fault location software is a function based program as shown in Fig. 4. There are three main functions which determine the fault location: -

i. The function getsamples() uses timer 2 interrupt service subroutine to generate a sampling period of $625 \mu$ s. At each sampling interval the function collects the instantaneous value of the line voltages and currents. It repeats this until the required number of samples is gathered.

ii. This data is passed on to the function DFT(), which extracts the amplitude and phase angle of the fundamental frequency.

iii. The analyse() function uses a fault current threshold determined during program initialisation to decide which phases are faulty. A variation of reactance equation is then applied to locate the fault depending on the number of faulty phases. The reactance equation gives a fault location in $m$ pu.

\section{EMTP-ATP Simulation}

A line-to-ground on phase A is simulated using an EMTP/ATP simulation model shown in Fig. 5. The fault occurs in the middle of the distribution line with a fault location of $0.5 \mathrm{pu}$. The EMTP/ATP model parameters are based on the laboratory power system model shown in Fig. 1.

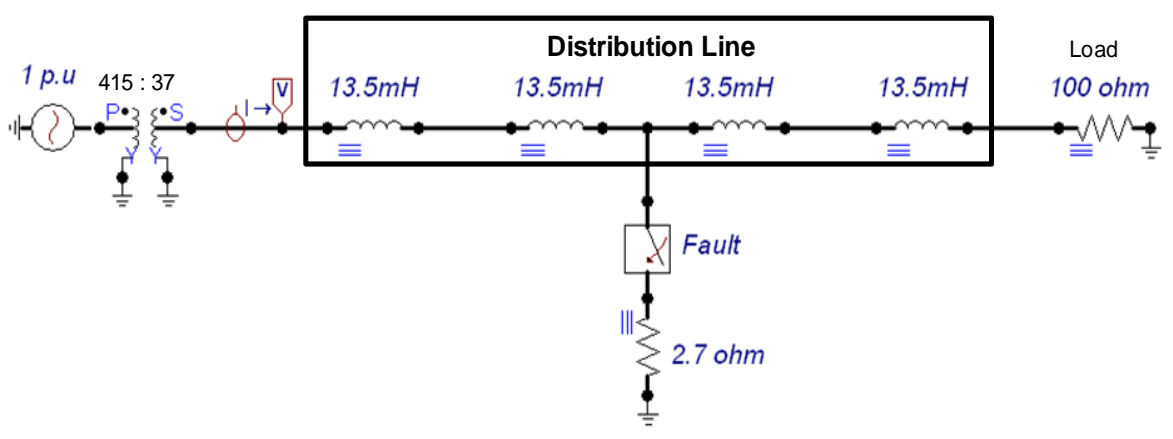

FIG. 5 ATP SIMULATION MODEL OF A FAULTY 415 V DISTRIBUTION LINE

\section{Results \& Discussion}

\section{EMTP-ATP Simulation}

A fault is set to occur at $t=0.06$ seconds and lasts for the remaining duration of the signal ( 5 cycles). The profiles of the ATP voltage and current waveforms can be seen for phases A, B and C in Fig. 6 and Fig. 7, respectively.

The DFT is used to extract the amplitude and phase angle of fundamental frequency line voltages and currents from Fig. 6 and Fig. 7. All transmission line signals are sampled at a rate of 32 samples per $50 \mathrm{~Hz}$ cycle, which produces a sampling period of $T_{s}=625 \mu \mathrm{s}$. The DFT fundamental frequency extraction of phase A for voltage and current can be seen in Fig. 8 and figure Fig. 9, respectively. By examining these waveforms it can be seen that there 
are 64 pre-fault samples (-64 to 0) and 160 samples after the time of fault occurrence. During the pre-fault samples, the fundamental frequency voltage and current amplitude is $1 \mathrm{pu}$. The application of a fault on the line causes the current at the sending end to increase to approximately $11 \mathrm{pu}$. Also, there is a decaying exponential DC offset of short duration. It should be noted that the DFT ignores this DC offset and just tracks the amplitude of the fundamental frequency, as required. The fundamental frequency voltage amplitude does not change by a significant amount.

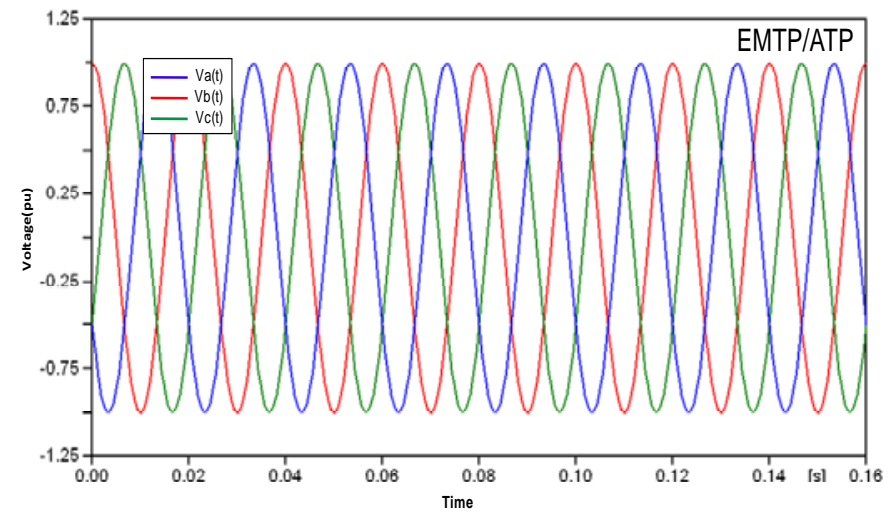

FIG. 6 ATP PHASE VOLTAGES WAVEFORMS

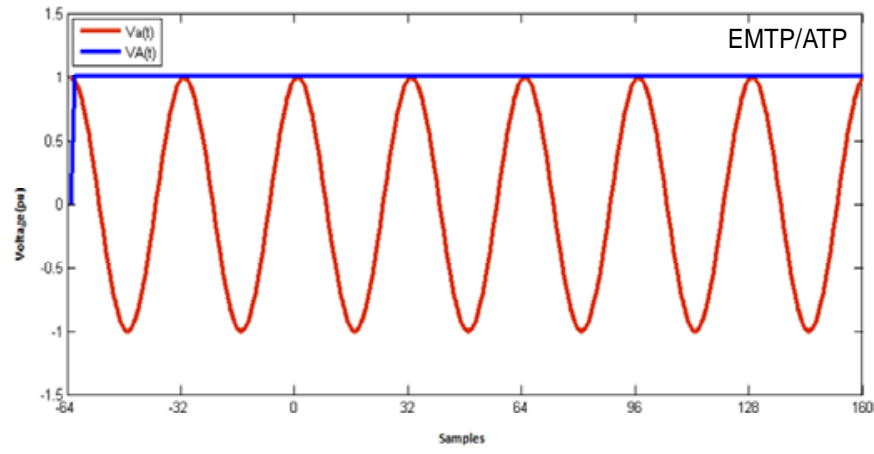

FIG. 8 EMTP-ATP FUNDAMENTAL FREQUENCY VOLTAGE EXTRACTION USING DFT

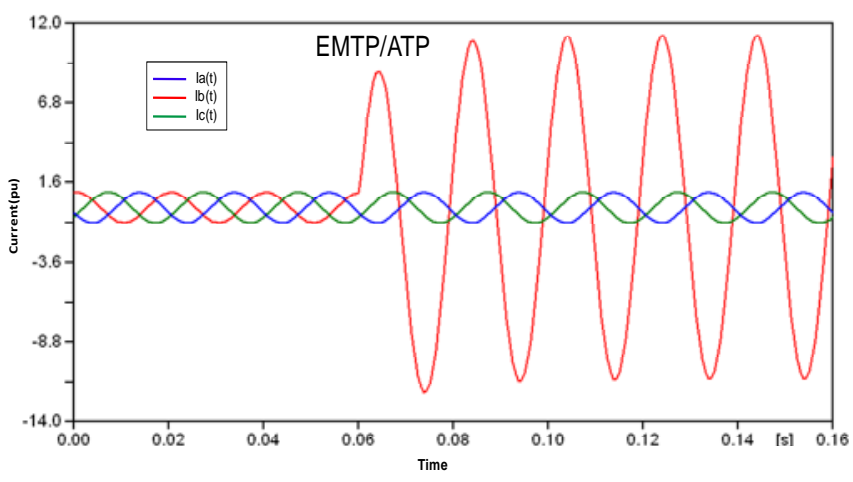

FIG. 7 ATP PHASE CURRENTS WAVEFORMS

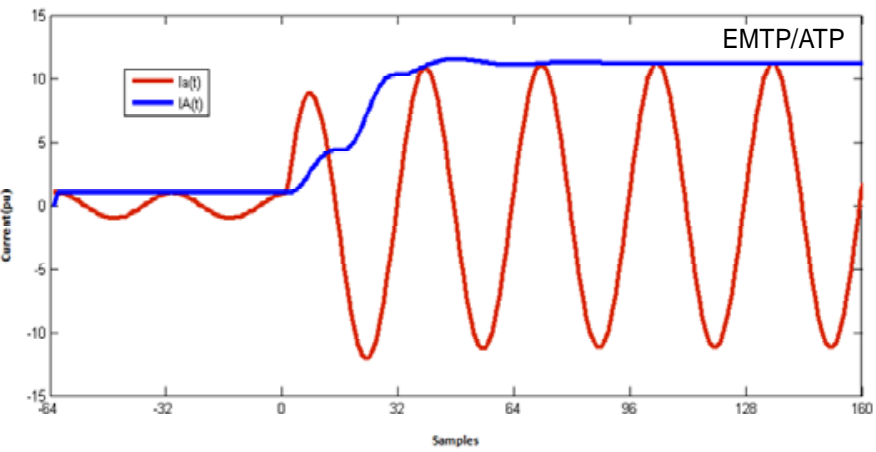

FIG. 9 EMTP-ATP FUNDAMENTAL FREQUENCY CURRENT EXTRACTION USING DFT

These results show that the DFT can extract the fundamental frequency in approximately 32 samples, i.e. a complete cycle of $50 \mathrm{~Hz}$. The resultant fault location in pu can be found by using the reactance equation (discussed earlier) as shown in Fig. 10.

This waveform correctly shows that the fault location converges to $0.5 \mathrm{pu}$ after the application of the fault.

The resultant fault location in per unit can be found using the reactance equation as show in Fig. 13.

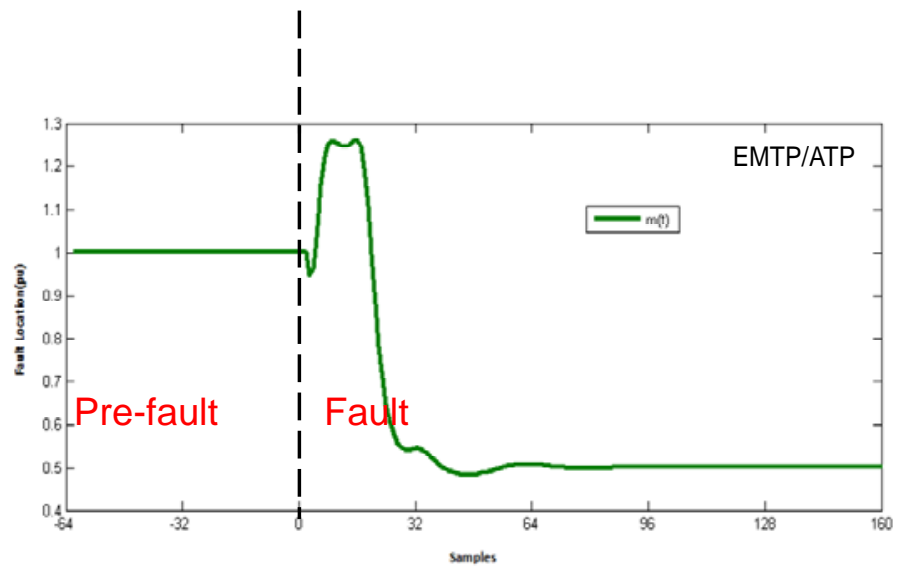

FIG. 10 EMTP-ATP FAULT LOCATION WAVEFORM

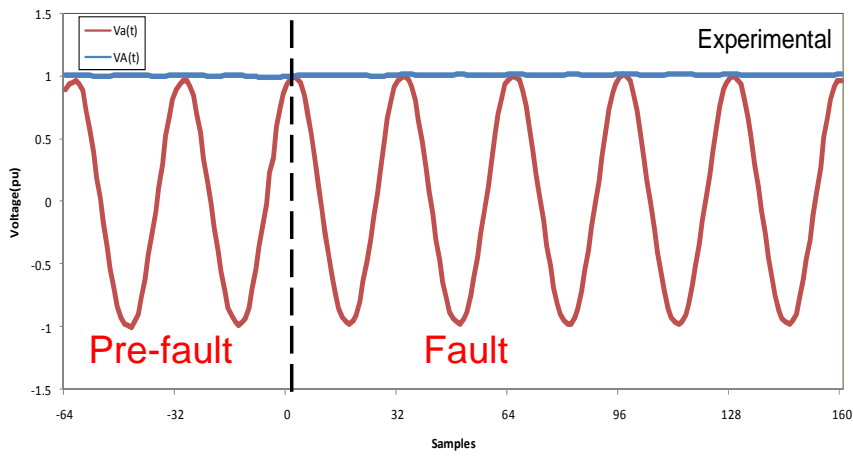

FIG. 11 MICROPROCESSOR BASED FUNDAMENTAL FREQUENCY VOLTAGE EXTRACTION USING DFT 


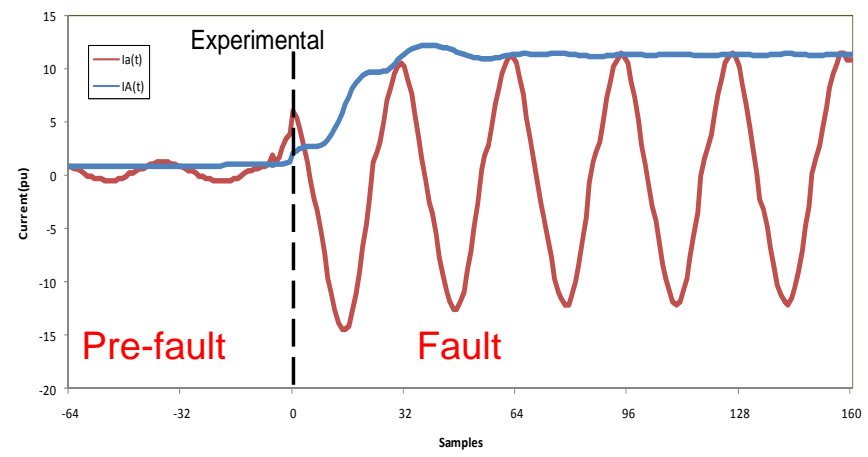

FIG. 12 MICROPROCESSOR BASED FUNDAMENTAL

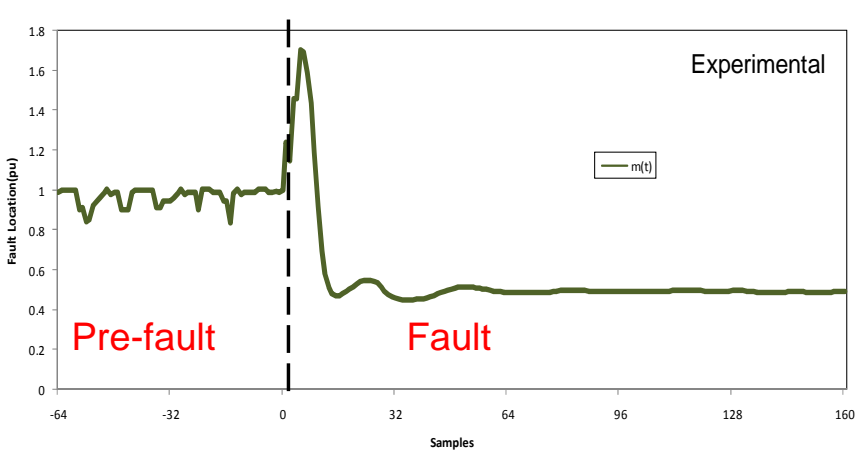

FIG. 13 MICROPROCESSOR FAULT LOCATION WAVEFORM FREQUENCY CURRENT EXTRACTION USING DFT

\section{Verification of Results Using the Microprocessor Based Fault Locator}

Results produced by EMTP/ATP simulation are verified using the microprocessor based fault locator on the laboratory power system model. The DFT is used to extract the fundamental frequency, amplitude and phase angle for all three line voltages and currents. Similarly to the EMTP/ATP simulation a sampling condition of 32 samples per $50 \mathrm{~Hz}$ cycle is used. The DFT fundamental frequency extraction of the voltage and current waveforms can be seen in Fig. 11 and Fig. 12, respectively.

\section{Conclusions}

The results produced in the EMTP/ATP environment are compared with experimental results produced by microprocessor based fault locator on a laboratory power system model. The DFT extracts the fundamental frequency in less than a complete cycle of $50 \mathrm{~Hz}$ and when combined with the reactance equation produces very accurate fault location. The DFT effectively ignores DC offset especially when present in heavily inductive lines.

A collapse in line voltage or an increase in line current is a useful indicator of a faulty phase. The fault locator allows quick detection for fast deactivation of the faulty line. This allows the power to be rerouted to the customer to meet target restoration times. Typically, distribution line faults have a fault impedance of unknown quantity, causing error in fault location. The reactance method significantly reduces this error to a tolerable amount. Generally, fault impedance is resistive in nature. The reactance equation ignores all resistances in the system and locates the fault purely based on reactive impedance.

The DFT requires a large amount of processing power due to the need for it to analyse a complete $50 \mathrm{~Hz}$ cycle at every sample period. It has the major advantage over other methods of extracting the fundamental frequency that no prior distribution line information is required apart from the reactive part of the line impedance.

\section{REFERENCES}

[1]. Croft, R. Davison, and M. Hargreaves, Engineering Mathematics: A Foundation for Electronic, Electrical, Communications, and Systems Engineers: Prentice Hall, 2001.

[2]. G. Phadke and J. S. Thorp, Computer relaying for power systems: John Wiley \& Sons, Inc. New York, NY, USA, 1988.

[3]. J. Wood and B. F. Wollenberg, Power generation, operation, and control: Wiley New York, 1984.

[4]. T. Johns and S. K. Salman, Digital Protection for Power Systems: Peter Peregrinus Ltd, 1995.

[5]. Wang, "Methodologies \& Algorithms for Fault Locators in Modern Power Systems," PhD Thesis, UWE Bristol, 2002.

[6]. F. H. Magnago and A. Abur, "Fault location using wavelets," Power Delivery, IEEE ransactions on, vol. 13, pp. 1475-1480, 1998.

[7]. H. Nouri and C. Wang, "A Fast Fault Classification Technique for Power Systems", International Journal of Advanced 
Research in Electrical, Electronics and Instrumentation Engineering, Vol. 3, Issue 11, November 2014.

[8]. H. Nouri, C. Wang, and T. Davies, "An Accurate Fault Location Technique for Distribution Lines With Tapped Loads Using Wavelet Transform," Proc. 2001 IEEE Power Tech, Porto, vol. 3, pp. 10-13, 2001.

[9]. K. Zimmerman and D. Costello, "Impedance-based fault location experience," Protective relay Engineers, 2005 58th Annual Conference for, pp. 211-226, 2005. 